TRANSACTIONS OF THE

AMERICAN MATHEMATICAL SOCIETY

Volume 352, Number 1, Pages 413-427

S 0002-9947(99)02183-2

Article electronically published on February 15, 1999

\title{
A LOCAL PETER-WEYL THEOREM
}

\author{
LEONARD GROSS
}

\begin{abstract}
An $A d K$ invariant inner product on the Lie algebra of a compact connected Lie group $K$ extends to a Hermitian inner product on the Lie algebra of the complexified Lie group $K_{c}$. The Laplace-Beltrami operator, $\Delta$, on $K_{c}$ induced by the Hermitian inner product determines, for each number $a>0$, a Green's function $r_{a}$ by means of the identity $\left(a^{2}-\Delta / 4\right)^{-1}=r_{a} *$. The Hilbert space of holomorphic functions on $K_{c}$ which are square integrable with respect to $r_{a}(x) d x$ is shown to be finite dimensional. It is spanned by the holomorphic extensions of the matrix elements of those irreducible representations of $K$ whose Casimir operator is appropriately related to $a$.
\end{abstract}

\section{INTRODUCTION}

Denote by $G$ a connected, simply connected complex Lie group. On its Lie algebra $\mathfrak{g}:=T_{e}(G)$ assume given a Hermitian inner product $(\cdot, \cdot)$. Choose an orthonormal basis $e_{1}, \ldots, e_{2 d}$ of $\mathfrak{g}$, regarded as a real vector space with inner product $\operatorname{Re}(\cdot, \cdot)$. Denoting by $\tilde{\xi}$ the left invariant vector field on $G$ which extends the element $\xi \in \mathfrak{g}$, define the Laplacian on $G$ associated to the given inner product as the second order differential operator $\Delta=\sum_{j=1}^{2 d}\left(\tilde{e}_{j}\right)^{2}$. This is in fact independent of the choice of orthonormal basis. The Laplacian has a self-adjoint version in $L^{2}(G, d x)$, wherein $d x$ denotes a right invariant Haar measure on $G$. There are two families of strictly positive functions on $G$ determined by the Laplacian: first there is the family of heat kernels, $\mu_{t}$, determined by the identity $e^{t \Delta / 4}=\mu_{t} *$; second there is the family of Green's functions, $r_{a}, a>0$, determined by the identity $\left(a^{2}-\Delta / 4\right)^{-1}=r_{a} *$. Write $\mathcal{H}(G)$ for the space of holomorphic functions on $G$. In [DG] the Hilbert space $\mathcal{H}(G) \cap L^{2}\left(G, \mu_{t}(x) d x\right)$ was analyzed with respect to the following question: since a holomorphic function $f$ is determined on all of $G$ by its family of Taylor "coefficients", i.e., derivatives, $\left\{D^{k} f(e)\right\}_{k=0}^{\infty}$, at the identity, how can the $L^{2}\left(G, \mu_{t}(x) d x\right)$ norm of $f$ be expressed in terms of these derivatives? In case $G=\mathbb{C}^{n}$ one has the classical result of Bargmann [B] which establishes a unitary map between the preceding Hilbert space, $\mathcal{H}\left(C^{n}\right) \cap L^{2}\left(\mathbb{C}^{n}\right.$, Gauss measure), and a Hilbert space of Taylor expansion coefficients. This isomorphism has been extended by Krée [K1], [K2], [K3] and Segal [S1], [S2], [S3] to infinite dimensions. In case $G$ is a general complex Lie group the analogous theorem was established in [DG]. The space of Taylor coefficients of functions in $\mathcal{H}(G) \cap L^{2}\left(G, \mu_{t}(x) d x\right)$ forms a Hilbert space which is more or less dual to the universal enveloping algebra of $\mathfrak{g}$. The main result of [DG] will be reviewed in Section 2 .

Received by the editors March 3, 1997 and, in revised form, April 18, 1997.

1991 Mathematics Subject Classification. Primary 22E30; Secondary 22C05.

This work was partially supported by NSF Grant DMS-9501238.

(c)1999 American Mathematical Society 
In this paper the heat kernels $\mu_{t}$ will be replaced by the Green's functions $r_{a}$. The Hilbert space $\mathcal{H}_{a}:=\mathcal{H}(G) \cap L^{2}\left(G, r_{a}(x) d x\right)$ will be shown to be isometrically isomorphic to a specific Hilbert space of Taylor coefficients. There is a surprising difference, however, from the heat kernel case: if $G$ is the complexification of a compact connected Lie group $K$, and the given inner product on $\mathfrak{g}$ restricts to an $A d K$ invariant inner product on LieK, then each Hilbert space $\mathcal{H}_{a}$ is finite dimensional. The dimension of $\mathcal{H}_{a}$ increases with $a$ and the jumps in the dimension are related to the dimensions of the irreducible unitary representations of $K$. In fact $\mathcal{H}_{a}$ is spanned by the matrix elements of those irreducible unitary representations whose Casimir operators are less than $a^{2}$. One obtains a kind of local Peter-Weyl theorem. A precise statement is given in Theorem 4.7.

\section{Notation AND BACKGROUND}

$G$ will denote a connected, complex Lie group (not necessarily simply connected). Its Lie algebra $\mathfrak{g}$ will be identified with $T_{e}(G)$. For any element $\xi$ in $\mathfrak{g}$ the left invariant extension of $\xi$ will be denoted by $\widetilde{\xi}$. Let $($,$) be a Hermitian inner$ product on $\mathfrak{g}$. If $e_{1}, \ldots, e_{2 d}$ is an orthonormal basis of $\mathfrak{g}$ as a real vector space and with respect to the real inner product $\operatorname{Re}($,$) , then the second order differential$ operator $\Delta$ defined by

$$
\Delta \varphi=\sum_{j=1}^{2 d}\left(\widetilde{e}_{j}\right)^{2} \varphi, \quad \varphi \in C_{c}^{\infty}(G),
$$

has a self-adjoint closure in $L^{2}(G, d x)$, where $d x$ denotes a right invariant Haar measure on $G$. The semigroup generated by $\Delta / 4$ is given by convolution with a probability density $\mu_{t}(x)$. That is,

$$
\left(e^{t \Delta / 4} f\right)(x)=\int_{G} f\left(x y^{-1}\right) \mu_{t}(y) d y, \quad f \in L^{2}(G, d x) .
$$

See [DG, Proposition 3.1] for further discussion of elementary properties of $\mu_{t}$. Denoting by $\mathcal{H}(G)$ the space of all holomorphic functions on $G$, define $\mathcal{H} L^{2}\left(G, \mu_{t}\right):=$ $\mathcal{H}(G) \cap L^{2}\left(G, \mu_{t}(x) d x\right)$. For any function $f$ in $\mathcal{H}(G)$ the $k$ th derivative functional $\left(D^{k} f\right)(x)$ at the point $x \in G$ is the unique element of $\left(\mathfrak{g}^{*}\right)^{\otimes k}$ defined by

$$
\left\langle\left(D^{k} f\right)(x), \xi_{1} \otimes \cdots \otimes \xi_{k}\right\rangle=\left(\widetilde{\xi}_{1} \cdots \widetilde{\xi}_{k} f\right)(x), \quad \xi_{j} \in \mathfrak{g}, j=1, \ldots, k, x \in G,
$$

where $\langle$,$\rangle denotes the complex bilinear pairing between \left(\mathfrak{g}^{*}\right)^{\otimes k}$ and $\mathfrak{g}^{\otimes k}$. Write also $\left(D^{0} f\right)(x)=f(x)$. Denote by $T(\mathfrak{g}):=\sum_{k=0}^{\infty} \mathfrak{g}^{\otimes k}$ (weak direct sum) the tensor algebra over $\mathfrak{g}$ and let $T^{\prime}(\mathfrak{g}):=\sum_{k=0}^{\infty}\left(\mathfrak{g}^{*}\right)^{\otimes k}$ (strong direct sum). $T^{\prime}(\mathfrak{g})$ is dual to $T(\mathfrak{g})$ in the pairing

$$
\langle\alpha, \beta\rangle=\sum_{k=0}^{\infty}\left\langle\alpha_{k}, \beta_{k}\right\rangle
$$

where

$$
\alpha=\sum_{k=0}^{\infty} \alpha_{k}, \quad \alpha_{k} \in\left(\mathfrak{g}^{*}\right)^{\otimes k}, k=0,1, \ldots
$$


and

$$
\beta=\sum_{k=0}^{N} \beta_{k}, \quad \beta_{k} \in \mathfrak{g}^{\otimes k}, \quad k=0,1, \ldots, N .
$$

For any function $f$ in $\mathcal{H}(G)$ the formal sum

$$
(1-D)_{e}^{-1} f=\sum_{k=0}^{\infty}\left(D^{k} f\right)(e), \quad f \in \mathcal{H}(G),
$$

always exists as an element of $T^{\prime}(\mathfrak{g})$. In the algebra $T(\mathfrak{g})$ let $J$ be the 2 -sided ideal generated by $\{\xi \otimes \eta-\eta \otimes \xi-[\xi, \eta]: \xi, \eta \in \mathfrak{g}\}$ and denote by $J^{0}$ its annihilator in $T^{\prime}(\mathfrak{g})$. It is an immediate consequence of the identity $[\xi, \eta]^{\sim}=[\widetilde{\xi}, \widetilde{\eta}]$ that $(1-D)_{e}^{-1} f$ lies in $J^{0}$. (See e.g. [DG, Sec. 2].) Moreover if, for $\xi \in \mathfrak{g}$, one writes $R_{\xi} \beta=\beta \otimes \xi$ for $\beta \in T(\mathfrak{g})$, then the right multiplication operator $R_{\xi}$ leaves the ideal $J$ invariant. The transpose $A_{\xi}:=R_{\xi}^{\text {transpose }}: T^{\prime}(\mathfrak{g}) \rightarrow T^{\prime}(\mathfrak{g})$ therefore carries $J^{0}$ into itself. The definitions (2.3) and (2.7) yield immediately the intertwining relation

$$
A_{\xi}(1-D)_{e}^{-1} f=(1-D)_{e}^{-1}(\widetilde{\xi} f), \quad f \in \mathcal{H}(G), \xi \in \mathfrak{g} .
$$

For each $t>0$ and each element $\alpha$ in $T^{\prime}(\mathfrak{g})$ write

$$
\|\alpha\|_{t}^{2}=\sum_{k=0}^{\infty}\left(t^{k} / k !\right)\left|\alpha_{k}\right|^{2}, \quad \alpha=\sum_{k=0}^{\infty} \alpha_{k}, \alpha_{k} \in\left(\mathfrak{g}^{*}\right)^{\otimes k} .
$$

Here $\left|\alpha_{k}\right|$ refers to the cross norm associated to the Hermitian inner product on $\left(\mathfrak{g}^{*}\right)^{\otimes k}$ induced by the given inner product on $\mathfrak{g}$. Define $J_{t}^{0}=\left\{\alpha \in J^{0}:\|\alpha\|_{t}<\infty\right\}$. Then $J_{t}^{0}$ is a complex Hilbert space. The main result in [DG] is summarized in the following theorem.

Theorem 2.1. [DG] The Taylor coefficient map $(1-D)_{e}^{-1}$ is an isometry from $\mathcal{H} L^{2}\left(G, \mu_{t}\right)$ into $J_{t}^{0}$. If, in addition, $G$ is simply connected, then $(1-D)_{e}^{-1}$ is unitary. Thus if $u$ is in $\mathcal{H}(G)$ and $\alpha=(1-D)_{e}^{-1} u$, then

$$
\sum_{k=0}^{\infty}\left(t^{k} / k !\right)\left|\alpha_{k}\right|^{2}=\int_{G}|u(x)|^{2} \mu_{t}(x) d x, \quad u \in \mathcal{H}(G) .
$$

\section{The Green's function Hilbert spaces}

Notation 3.1. Let $a>0$. define

$$
r_{a}(x)=\int_{0}^{\infty} e^{-t a^{2}} \mu_{t}(x) d t, \quad x \in G .
$$

Since $\int_{G} \mu_{t}(x) d x=1$ we find, applying Fubini's theorem, that

$$
\int_{G} r_{a}(x) d x=a^{-2}
$$

Convolution by $r_{a}$ is therefore a bounded operator in $L^{2}(G, d x)$. Multiplying Equation $(2.2)$ by $e^{-t a^{2}}$ and integrating over $(0, \infty)$ yields, informally,

$$
\left(\left(a^{2}-\Delta / 4\right)^{-1} f\right)(x)=\int_{G} f\left(x y^{-1}\right) r_{a}(y) d y, \quad f \in L^{2}(G, d x) .
$$

This equation is easily justified by verifying it first for nonnegative functions $f$ in $C_{c}(G)$ and interpreting the integral over $t$ on the left as a weak integral. However 
Equation (3.3) will not be used in any technical way itself. The Green's function norms will be denoted by

$$
\|u\|_{, a}^{2}=\int_{G}|u(x)|^{2} r_{a}(x) d x, \quad a>0,
$$

and the Green's function Hilbert spaces are

$$
\mathcal{H}_{a}=\mathcal{H}(G) \cap L^{2}\left(G, r_{a}(x) d x\right) .
$$

Now suppose that $u \in \mathcal{H}(G)$ and $\alpha=(1-D)_{e}^{-1} u$. Multiply Equation (2.10) by $e^{-t a^{2}}$ and integrate with respect to $t$ over $(0, \infty)$. One finds, using Fubini's theorem on both sides,

$$
\sum_{k=0}^{\infty}\left(a^{2}\right)^{-k-1}\left|\alpha_{k}\right|^{2}=\int_{G}|u(x)|^{2} r_{a}(x) d x, \quad u \in \mathcal{H}(G), a>0 .
$$

With this computation as motivation define now

$$
\|\alpha\|_{, a}^{2}=\sum_{k=0}^{\infty}\left(a^{2}\right)^{-k-1}\left|\alpha_{k}\right|^{2}, \quad \alpha=\sum_{k=0}^{\infty} \alpha_{k}, \quad \alpha_{k} \in\left(\mathfrak{g}^{*}\right)^{\otimes k}, k=0,1, \ldots
$$

The Taylor coefficient space associated to this norm is

$$
J_{, a}^{0}=\left\{\alpha \in J^{0}:\|\alpha\|_{, a}<\infty\right\} .
$$

The Equation (3.6) shows that if $u$ is in $\mathcal{H}_{a}$, then $(1-D)_{e}^{-1} u$ is in $J_{, a}^{0}$. Note also that $J_{, a}^{0}$ is complete because for any element $j \in J$ the map $\alpha \rightarrow{ }_{T^{\prime}(\mathfrak{g})}\langle\alpha, j\rangle_{T(\mathfrak{g})}$ is continuous in \|\|$_{, a}$ norm.

Proposition 3.2. The map $u \rightarrow(1-D)_{e}^{-1} u$ is isometric from $\mathcal{H}_{a}$ into $J_{, a}^{0}$. Moreover

$$
J_{, a}^{0} \subset J_{, b}^{0} \subset \bigcap_{t>0} J_{t}^{0}, \quad 0<a<b,
$$

and

$$
\mathcal{H}_{a} \subset \mathcal{H}_{b} \subset \bigcap_{t>0} \mathcal{H} L^{2}\left(G, \mu_{t}\right), \quad 0<a<b .
$$

If in addition $G$ is simply connected, then $(1-D)_{e}^{-1}$ is unitary.

Proof. Equation (3.6) shows that $(1-D)_{e}^{-1}: \mathcal{H}_{a} \rightarrow J_{, a}^{0}$ is isometric. Since $t^{k} / k !=$ $o\left(\left(b^{2}\right)^{-k-1}\right)$ as $k \rightarrow \infty$ and $\left(b^{2}\right)^{-k-1}=o\left(\left(a^{2}\right)^{-k-1}\right)$ as $k \rightarrow \infty$ the containments (3.9) follow from (3.7) and (2.9). The containments (3.10) now follow from (3.6) and (2.10). If $G$ is simply connected and $\alpha \in J_{, a}^{0}$, then by (3.9) $\alpha$ is in $J_{t}^{0}$ for some (actually all) $t>0$. By Theorem 2.1 there exists a function $u$ in $\mathcal{H}(G)$ such that $\alpha=(1-D)_{e}^{-1} u$. Equation (3.6) shows that $u \in \mathcal{H}_{a}$. Q.E.D.

The procedure used in deriving Equation (3.6) from Equation (2.10) can be used to derive other identities which also express some $L^{2}$ norm of a holomorphic function $u$ in terms of its derivatives at the identity element. Suppose that $h$ is a nonnegative integrable function on $[0, \infty)$. Let $\psi(s)=\int_{0}^{\infty} h(t) e^{-t s} d t$. Then the operator $\psi(-\Delta / 4)$ is given by convolution by a positive finite measure $\tau$. Multiplying Equation $(2.10)$ by $h(t)$ and integrating over $[0, \infty)$ as above gives an identity similar to (3.6), with the measure $r_{a}(x) d x$ on the right replaced by $\tau$ and the coefficient of $\left|\alpha_{k}\right|^{2}$ on the left replaced by a $k$ th moment of $h$. We will not have occasion to use these more general identities here. 
The real inner product $\operatorname{Re}(\cdot, \cdot)$ on $\mathfrak{g}$ extends by left invariance to a Riemannian metric on $G$. Denote the associated (left invariant) distance function on $G$ by $d(x, y)$ and write $|x|=d(e, x)$ for $x$ in $G$.

Proposition 3.3 (Pointwise bounds). Let $u$ be in $\mathcal{H}_{a}$. Then

$$
|u(x)| \leq a\|u\|_{, a} e^{a|x|}, \quad u \in \mathcal{H}_{a} .
$$

Proof. The same method used in [DG, Proposition 3.9] to bound derivatives of $u$ when $u \in \mathcal{H} L^{2}\left(G, \mu_{t}\right)$ applies in the present context with some slight modifications. Let $\sigma:[0,1] \rightarrow G$ be a smooth curve in $G$ with $\sigma(0)=e$ and $\sigma(1)=x$. Let $c(s)=\left(L_{\sigma(s)^{-1}}\right)_{*} \sigma^{\prime}(s)$ be the left translate of the tangent vector $\sigma^{\prime}(s)$ back to the identity. Define

$$
\psi(\sigma)=\sum_{n=0}^{\infty} \int_{\Delta_{n}} c\left(s_{1}\right) \otimes \cdots \otimes c\left(s_{n}\right) d \mathbf{s}
$$

where $\Delta_{n}=\left\{\left(s_{1}, \ldots, s_{n}\right): 0<s_{1}<\cdots<s_{n}<1\right\}$ and $d \mathbf{s}=d s_{1} \cdots d s_{n}$. The global recovery formula for $u$ from its Taylor coefficients is given by the generalized power series

$$
u(x)=\langle\alpha, \psi(x)\rangle, \quad \alpha=(1-D)_{e}^{-1} u .
$$

See Proposition 5.1 of [D] for an efficient derivation of this identity. The norm on $T(\mathfrak{g})$ dual to that given in $(3.7)$ is

$$
\|\beta\|_{, a}^{2}=\sum_{k=0}^{\infty}\left(a^{2}\right)^{k+1}\left|\beta_{k}\right|^{2}, \beta=\sum_{k=0}^{\infty} \beta_{k}, \beta_{k} \in \mathfrak{g}^{\otimes k}, k=0,1, \ldots
$$

We will see immediately that $\psi(\sigma)$ is in the completion of $T(\mathfrak{g})$ in this norm so that the series on the right of (3.13) converges. We have

$$
|u(x)| \leq\|\alpha\|_{, a}\|\psi(\sigma)\|_{, a}=\|u\|_{, a}\|\psi(\sigma)\|_{, a}
$$

where $\|\psi(\sigma)\|_{, a}$ is given by (3.14). Let $\rho=\int_{0}^{1}|c(s)| d s$. Then

$$
\begin{aligned}
\left|\int_{\Delta_{n}} c\left(s_{1}\right) \otimes \cdots \otimes c\left(s_{n}\right) d \mathbf{s}\right|^{2} & =\int_{\Delta_{n} \times \Delta_{n}} \prod_{j=1}^{n}\left(c\left(s_{j}\right), c\left(t_{j}\right)\right) d \mathbf{s} d \mathbf{t} \\
& \leq \int_{\Delta_{n} \times \Delta_{n}} \prod_{j=1}^{n}\left|c\left(s_{j}\right)\right|\left|c\left(t_{j}\right)\right| d \mathbf{s} d \mathbf{t} \\
& =\rho^{2 n} /(n !)^{2} .
\end{aligned}
$$

Hence

$$
\|\psi(\sigma)\|_{, a}^{2} \leq a^{2} \sum_{k=0}^{\infty}\left(a^{2}\right)^{k} \rho^{2 k} /(k !)^{2}=a^{2} v(a \rho)
$$

where $v(s)=\sum_{k=0}^{\infty} s^{2 k} /(k !)^{2}$. We will show in the next lemma that $v(s) \leq e^{2 s}$ for $s \geq 0$. Using this inequality in combination with (3.15) and (3.17) one finds $|u(x)| \leq a\|u\|_{, a} e^{a \rho}$. Since, by definition, $|x|=\inf \{\rho: \sigma(1)=x\}$ the inequality (3.11) follows. Q.E.D. 
Lemma 3.4. Let $v(s)=\sum_{k=0}^{\infty} s^{2 k} /(k !)^{2}$. Then $v(s) \leq e^{2 s}$ for $s \geq 0$.

Proof. A proof of this lemma for the modified Bessel function $v$ could be deduced from known integral formulas for $v$. But here is an elementary proof. For $s \geq 0$ we have

$$
\begin{aligned}
v^{\prime}(s) & =2 \sum_{k=1}^{\infty}\left(s^{k-1} /(k-1) !\right)\left(s^{k} / k !\right) \\
& \leq 2\left(\sum_{k=1}^{\infty} s^{2(k-1)} /((k-1) !)^{2}\right)^{1 / 2}\left(\sum_{k=1}^{\infty} s^{2 k} /(k !)^{2}\right)^{1 / 2} \\
& \leq 2 v(s) .
\end{aligned}
$$

So $(d / d s) \log v(s) \leq 2$. Since $\log v(0)=0$ we find $\log v(s) \leq 2 s$. Q.E.D.

Proposition 3.5. As an operator on $J_{, a}$ the annihilation operator $A_{\xi}$ (see Section 2) is bounded for each $\xi$ in $\mathfrak{g}$. Moreover

$$
\left\|A_{\xi}\right\| \leq a|\xi| \text {. }
$$

Correspondingly, the differential operators $\widetilde{\xi}$ are bounded on $\mathcal{H}_{a}$ with

$$
\|\widetilde{\xi} u\|_{, a} \leq a|\xi|\|u\|_{, a}, \quad u \in \mathcal{H}_{a} .
$$

Furthermore

$$
\||D u|\|_{, a}^{2}=a^{2}\|u\|_{, a}^{2}-|u(e)|^{2}=a^{2}\|u-u(e)\|_{, a}^{2} .
$$

Proof. Let $e_{1}, \ldots, e_{d}$ be an orthonormal basis of $\mathfrak{g}$ as a complex inner product space. Let $f_{1}, \ldots, f_{d}$ be the dual basis of $\mathfrak{g}^{*}$. For $k \geq 1$ any element $\alpha$ of $\left(\mathfrak{g}^{*}\right)^{\otimes k}$ is uniquely of the form $\alpha=\sum_{j=1}^{d} \alpha_{j} \otimes f_{j}$ with $\alpha_{j}$ in $\left(\mathfrak{g}^{*}\right)^{\otimes k-1}$. It follows from the definition of $A_{\xi}$ that $A_{\xi} \alpha=\sum_{j=1}^{d} \alpha_{j}\left\langle f_{j}, \xi\right\rangle$. In particular $A_{e_{n}} \alpha=\alpha_{n}$. Hence

$$
\sum_{j=1}^{d}\left|A_{e_{j}} \alpha\right|^{2}=\sum_{j=1}^{d}\left|\alpha_{j}\right|^{2}=|\alpha|^{2} .
$$

For any element $\alpha$ in $T^{\prime}(\mathfrak{g})$ we therefore have (cf. (3.7))

$$
\begin{aligned}
\sum_{j=1}^{d}\left\|A_{e_{j}} \alpha\right\|_{, a}^{2} & =\sum_{k=0}^{\infty}\left(a^{2}\right)^{-k-1} \sum_{j=1}^{d}\left|\left(A_{e_{j}} \alpha\right)_{k}\right|^{2} \\
& =\sum_{k=0}^{\infty}\left(a^{2}\right)^{-k-1}\left|\alpha_{k+1}\right|^{2} \\
& =a^{2} \sum_{k=0}^{\infty}\left(a^{2}\right)^{-(k+1)-1}\left|\alpha_{k+1}\right|^{2} \\
& =a^{2}\|\alpha\|_{, a}^{2}-\left|\alpha_{0}\right|^{2} \\
& \leq a^{2}\|\alpha\|_{, a}^{2} .
\end{aligned}
$$


In particular $\left\|A_{e_{1}} \alpha\right\|_{, a}^{2} \leq a^{2}\|\alpha\|_{, a}^{2}$. Thus if $\xi$ is not zero and $e_{1}$ is chosen to be $\xi /|\xi|$, then (3.18) follows. Since $(1-D)_{e}^{-1}$ is isometric and intertwines $\widetilde{\xi}$ and $A_{\xi},(3.18)$ implies (3.19). (If $G$ is simply connected, then (3.18) and (3.19) are equivalent since $(1-D)_{e}^{-1}$ is then unitary.) Now $\|D u\|_{, a}^{2}=\sum_{j=1}^{d}\left\|\widetilde{e}_{j} u\right\|_{, a}^{2}=\sum_{j=1}^{d}\left\|A_{e_{j}} \alpha\right\|_{, a}^{2}$. Since $\alpha_{0}=u(e)$ the next to the last line in (3.21) yields the first equality in (3.20). But since $D(u-u(e))=D u$, the first equality in (3.20), applied to $u-u(e)$, yields the second equality. Q.E.D.

Remark 3.6. It is illuminating to see how a derivation of the identity (3.20) looks on the function side rather than on the Taylor expansion side. Since we already have a precise proof, let us proceed informally using integration by parts without justification. The Green's kernel $r_{a}$ is the fundamental solution to the equation $\left(\left(a^{2}-\Delta / 4\right) r_{a}\right)(x)=\delta(x)$. Thus $(\Delta / 4) r_{a}(x)=a^{2} r_{a}(x)-\delta(x)$. Now if $u \in \mathcal{H}(G)$, then $|D u(x)|^{2}=(\Delta / 4)|u(x)|^{2}$. (See e.g. [DG, Remark 3.7].) Hence

$$
\begin{aligned}
\int|D u(x)|^{2} r_{a}(x) d x & =\int\left\{(\Delta / 4)|u(x)|^{2}\right\} r_{a}(x) d x=\int|u(x)|^{2}(\Delta / 4) r_{a}(x) d x \\
& =a^{2} \int|u(x)|^{2} r_{a}(x) d x-\int|u(x)|^{2} \delta(x) d x
\end{aligned}
$$

which is (3.20).

The following theorem summarizes the principle properties of the Green's function Hilbert spaces that have been proven above.

Theorem 3.7. a) The Taylor coefficient map $(1-D)_{e}^{-1}: \mathcal{H}_{a} \rightarrow J_{, a}^{0}$ is isometric. If $G$ is simply connected, it is unitary.

b) (Pointwise bounds) If $u$ is in $\mathcal{H}_{a}$, then it has at most exponential growth given by (3.11).

c) (Annihilation operators are bounded) The annihilation operators $A_{\xi}: J_{, a}^{0} \rightarrow$ $J_{, a}^{0}$ are bounded for $\xi$ in $\mathfrak{g}$ with bounds given by (3.18) while the left invariant vector fields $\widetilde{\xi}: \mathcal{H}_{a} \rightarrow \mathcal{H}_{a}$ are also bounded with bounds given by (3.19).

Example 3.8. Let $G=\mathbb{C}^{n}$. In this case

$$
\mu_{t}(z)=(\pi t)^{-n} e^{-|z|^{2} / t}, \quad z \in \mathbb{C}^{n} .
$$

The asymptotic behavior of $r_{a}(z)$ as $|z| \rightarrow \infty$ can be obtained from (3.1). Insert (3.22) into (3.1) and make the substitution $t=(|z| / a) s$. One finds

$$
r_{a}(z)=(a /|z|)^{n-1} \int_{0}^{\infty} e^{-a|z|\left(s+s^{-1}\right)}(\pi s)^{-n} d s .
$$

By standard asymptotic expansion methods (see e.g. [BO, Chapter 6]) one obtains

$$
\int_{0}^{\infty} e^{-x\left(s+s^{-1}\right)}(\pi s)^{-n} d s \cong x^{-1 / 2} e^{-2 x} \pi^{-(n+1 / 2)} \quad \text { as } \quad x \rightarrow+\infty,
$$

in the sense that the ratio approaches one as $x \rightarrow+\infty$. In combination with (3.23) this yields

$$
r_{a}(z) \cong(a /|z|)^{n-(1 / 2)} e^{-2 a|z|} a^{-1} \pi^{-n-(1 / 2)} \quad \text { as } \quad|z| \rightarrow \infty
$$

in the sense that the ratio approaches one as $|z| \rightarrow \infty$. Notice that the pointwise growth bound given in (3.11), $|u(z)|^{2} \leq$ const. $e^{2 a|z|}$, is consistent with (3.24) in 
the sense that (3.11) describes the largest exponential growth rate consistent with square integrability of $u$. There are two interesting conclusions to be observed in this example. First, each space $\mathcal{H}_{a}\left(\mathbb{C}^{n}\right)$ is infinite dimensional because it contains all polynomials (as well as the exponentials $u(z)=e^{\gamma \cdot z}$ with $\gamma \in \mathbb{C}^{n}$ and $|\gamma|<$ $a$ ). Second, for $0<a<b, \mathcal{H}_{b}(\mathbb{C})$ is strictly larger than $\mathcal{H}_{a}(\mathbb{C})$. (Consider the exponentials $e^{\gamma z}$ with $a<\gamma<b$.) In Section 5 it will be shown that both of these conclusions fail in case $G=S L(2, \mathbb{C})$.

The singularity at $z=0$ which one sees in (3.23) is typical of Green's functions. It will be convenient for application in the next section to replace the singular density $r_{a}$ on $G$ by a smooth density. Of course the full $L^{2}$ spaces for two such different densities cannot be the same. But the following proposition shows that on $\mathcal{H}(G)$ one can have equivalence of norms.

Proposition 3.9. Let $T>0$. Define

$$
r_{a}^{T}(x)=\int_{T}^{\infty} e^{-t a^{2}} \mu_{t}(x) d x, \quad x \in G, a>0 .
$$

Then

$$
\begin{aligned}
e^{-a^{2} T} \int_{G}|u(x)|^{2} r_{a}(x) d x & \leq \int_{G}|u(x)|^{2} r_{a}^{T}(x) d x \\
& \leq \int_{G}|u(x)|^{2} r_{a}(x) d x, \quad u \in \mathcal{H}(G) .
\end{aligned}
$$

Proof. For any $c \geq 0$ and $k \geq 0$ let $w_{k}(c, T)=e^{-c T} \sum_{j=0}^{k}(c, T)^{j} / j !$. Then

$$
e^{-c T} \leq w_{k}(c T) \leq 1 \text {. }
$$

Moreover one can compute easily that $(\partial / \partial T)\left(k ! / c^{k+1}\right) w_{k}(c, T)=-T^{k} e^{c T}$. Hence $\int_{T}^{\infty} t^{k} e^{-c t} d t=\left(k ! / c^{k+1}\right) w_{k}(c, T)$ (plus a constant which is zero because both sides $\rightarrow 0$ as $T \rightarrow \infty)$. Multiply Equation $(2.10)$ by $e^{-a^{2} t}$ and integrate from $t=T$ to infinity to find

$$
\sum_{k=0}^{\infty}\left(a^{2}\right)^{-k-1} w_{k}\left(a^{2}, T\right)\left|\alpha_{k}\right|^{2}=\int_{G}|u(x)|^{2} r_{a}^{T}(x) d x .
$$

Combining (3.6), (3.28) and (3.27) (with $c=a^{2}$ ) yields (3.26). Q.E.D.

\section{The Green's function Hilbert spaces FOR THE COMPLEXIFICATION OF A COMPACT GROUP}

Let $K$ be a compact connected Lie group. In this section $G$ will be taken to be the complexification of $K$. See [H] for a discussion of complexification of a compact Lie group. Several results and techniques from $[\mathrm{H}]$ will be used in this section. We assume given on the Lie algebra $\mathfrak{k}$, which we will identify with $T_{e}(K)$, an $\operatorname{Ad} K$ invariant inner product $\langle$,$\rangle . Since \mathfrak{g}=\mathfrak{k} \otimes \mathbb{C}$ the inner product $\langle$,$\rangle has a unique$ extension ( , ) to a Hermitian inner product on $\mathfrak{g}$. In this section this inner product will be used to construct the Hilbert spaces $\mathcal{H}_{a}$ and $J_{, a}^{0}$ discussed in Section 3 . 
Theorem 4.1. Let $a>0$. There exists a constant $C$ depending on a such that

$$
\begin{array}{r}
\int_{G}\left|u\left(k_{2}^{-1} g k_{1}\right)\right|^{2} r_{a}(g) d g \leq C \int_{G}|u(g)|^{2} r_{a}(g) d g, \text { for all } u \in \mathcal{H}(G), \\
\text { and all } k_{1} \text { and } k_{2} \text { in } K .
\end{array}
$$

We will need the following lemma of Hall $[\mathrm{H}]$.

Lemma 4.2 (Hall's averaging lemma). Let $T>0$ and write $d k$ for normalized Haar measure on $K$. Define

$$
\nu_{t}(g)=\int_{K} \mu_{t}(k g) d k, \quad g \in G, t>0 .
$$

Then there are strictly positive constants $C_{1}$ and $C_{2}$ such that

$$
C_{1} \nu_{t}(g) \leq \mu_{t}(g) \leq C_{2} \nu_{t}(g) \forall g \in G \text { and } \forall t \geq T \text {. }
$$

Proof. This differs from the statement of Lemma 11 in $[\mathrm{H}]$ only in the assertion that the constants $C_{1}$ and $C_{2}$ can be taken to be independent of $t$ in the interval $[T, \infty)$. But an inspection of Hall's proof shows that such independence of $t$ will follow if one can show that there exist constants $a$ and $b$ such that $0<a \leq \rho_{s}(x) \leq b$ for all $x$ in $K$ and all $s \geq T / 4$ where $\rho_{s}$ is the heat kernel for $K$. Such constants exist for fixed $s$, say $s=T / 4$, because $\rho_{T / 4}$ is continuous and strictly positive on $K$. But if $s>T / 4$, say $s=r+T / 4$, then the semigroup equation, $\rho_{s}(x)=\int_{K} \rho_{r}\left(x y^{-1}\right) \rho_{T / 4}(y) d y$, shows that the same constants $a$ and $b$ work for all $s \geq T / 4$. Q.E.D.

Proof of Theorem 4.1. Choose $T>0$. Define a $K$ averaged, truncated Green's function $\widehat{r}_{a}^{T}$ on $G$ by

$$
\widehat{r}_{a}^{T}(g)=\int_{T}^{\infty} e^{-a^{2} t} \nu_{t}(g) d t, \quad g \in G .
$$

Then by the definition (3.25) and the inequalities (4.3) we have

$$
C_{1} \widehat{r}_{a}^{T}(g) \leq r_{a}^{T}(g) \leq C_{2} \widehat{r}_{a}^{T}(g), \quad g \in G
$$

Since the Laplacian, $\Delta$, on $G$ is invariant under the adjoint action of $K$, the heat kernel density $\mu_{t}$ is invariant under $K$ in the sense that $\mu_{t}\left(k^{-1} g k\right)=\mu_{t}(g)$ for all $g$ in $G$ and all $k$ in $K$. It is clear from (4.2) that $\nu_{t}$ is left $K$ invariant. But it is also right $K$ invariant on $G$ because if $m \in K$, then

$$
\begin{aligned}
\nu_{t}(g m) & =\int_{K} \mu_{t}(k g m) d k=\int_{K} \mu_{t}\left(m^{-1} m k g m\right) d k=\int_{K} \mu_{t}(m k g) d k \\
& =\int_{K} \mu_{t}(k g) d k=\nu_{t}(g) .
\end{aligned}
$$

Therefore (4.4) shows that $\widehat{r}_{a}^{T}(g)$ is also left and right $K$ invariant. Suppose, then, that $u \in \mathcal{H}(G)$. Using both inequalities in (3.26) and both inequalities in (4.5) we 
find, for any elements $k_{1}$ and $k_{2}$ in $K$,

$$
\begin{aligned}
\int_{G}\left|u\left(k_{2}^{-1} g k_{1}\right)\right|^{2} r_{a}(g) d g & \leq e^{a^{2} T} \int_{G}\left|u\left(k_{2}^{-1} g k_{1}\right)\right|^{2} r_{a}^{T}(g) d g \\
& \leq e^{a^{2} T} C_{2} \int_{G}\left|u\left(k_{2}^{-1} g k_{1}\right)\right|^{2} \widehat{r}_{a}^{T}(g) d g \\
& =e^{a^{2} T} C_{2} \int_{G}|u(g)|^{2} \widehat{r}_{a}^{T}\left(k_{2} g k_{1}^{-1}\right) d g \\
& =e^{a^{2} T} C_{2} \int_{G}|u(g)|^{2} \widehat{r}_{a}^{T}(g) d g \\
& \leq e^{a^{2} T} C_{2} C_{1}^{-1} \int_{G}|u(g)|^{2} r_{a}^{T}(g) d g \\
& \leq e^{a^{2} T} C_{2} C_{1}^{-1} \int_{G}|u(g)|^{2} r_{a}(g) d g . \quad \text { Q.E.D. }
\end{aligned}
$$

Denote now by $\widehat{K}$ the set of isomorphism classes of irreducible unitary representations of $K$. If $u \in \mathcal{H}(G)$, then the restriction of $u$ to $K$ is a smooth function on $K$ and is therefore given by its Fourier series

$$
u(k)=\sum_{\pi \in \widehat{K}} \operatorname{tr}\left(\pi(k) A_{\pi}\right), \quad k \in K,
$$

where $A_{\pi} \in \operatorname{End}\left(V_{\pi}\right)$ and $V_{\pi}$ denotes the finite dimensional inner product space on which $\pi(k)$ acts. Each representation $\pi$ in $\widehat{K}$ extends holomorphically to a homomorphism $\pi: G \rightarrow \operatorname{End}\left(V_{\pi}\right)$. It is shown in [H, Lemma 9] that if $u$ is given on $K$ by (4.6), then $u$ is given on $G$ by the series

$$
u(g)=\sum_{\pi \in \widehat{K}} \operatorname{tr}\left(\pi(g) A_{\pi}\right), \quad g \in G,
$$

and that this series converges uniformly on compact subsets of $G$.

Corollary 4.3. Let $a>0$. Suppose that $u \in \mathcal{H}_{a}(G)$ and that $u$ is given by the series (4.7). Then each term, $\operatorname{tr}\left(\pi(\cdot) A_{\pi}\right)$, is also in $\mathcal{H}_{a}(G)$.

Proof. By Theorem 4.1 there is a constant $C$, independent of $k$, such that

$$
\int_{G}\left|u\left(k^{-1} g\right)\right|^{2} r_{a}(g) d g \leq C\|u\|_{, a}^{2}, \quad k \in K .
$$

We may integrate both sides over $K$ and use the Peter-Weyl theorem to find

$$
\sum_{\pi \in \widehat{K}}\left(\operatorname{dim} V_{\pi}\right)^{-1} \int_{G} \operatorname{tr}\left\{\left(\pi(g) A_{\pi}\right)^{*}\left(\pi(g) A_{\pi}\right)\right\} r_{a}(g) d g \leq C\|u\|_{, a}^{2} .
$$

Since each summand in this inequality is non-negative, each integral is finite. Hence each term $\operatorname{tr}\left(\pi(g) A_{\pi}\right)$ in (4.7) is in $\mathcal{H}_{a}$. Q.E.D.

Corollary 4.4. Let $a>0$ and suppose that $\pi$ is an irreducible unitary representation of $K$. If $\operatorname{tr}(\pi(\cdot) A) \in \mathcal{H}_{a}(G)$ for some nonzero operator $A$ in $\operatorname{End}\left(V_{\pi}\right)$, then $\operatorname{tr}(\pi(\cdot) A)$ is in $\mathcal{H}_{a}$ for all $A$ in $\operatorname{End}\left(V_{\pi}\right)$.

Proof. Write $u(g)=\operatorname{tr}(\pi(g) A)$. By assumption $u \in \mathcal{H}_{a}$. By Theorem $4.1 u\left(k_{2}^{-1} \cdot k_{1}\right)$ is also in $\mathcal{H}_{a}$ for all $k_{1}$ and $k_{2}$ in $K$. That is, the function $g \rightarrow \operatorname{tr}\left(\pi(g) \pi\left(k_{1}\right) A \pi\left(k_{2}^{-1}\right)\right)$ is in $\mathcal{H}_{a}$. But since $\pi \mid K$ is irreducible the $\operatorname{set}\left\{\pi\left(k_{1}\right) A \pi\left(k_{2}^{-1}\right): k_{1}, k_{2} \in K\right\}$ is 
fundamental in $\operatorname{End}\left(V_{\pi}\right)$. (To see this identify $\operatorname{End}\left(V_{\pi}\right)$ with $V_{\pi} \otimes V_{\pi}^{*}$ and use the irreducibility of $\left.K \times K \ni\left(k_{1}, k_{2}\right) \rightarrow \pi\left(k_{1}\right) \otimes \pi\left(k_{2}^{-1}\right)^{\text {transpose }}.\right)$ Q.E.D.

Notation 4.5. If $\pi$ is an irreducible representation of $K$ in $V_{\pi}$ and $e_{1}, \ldots, e_{d}$ is an orthonormal basis of $\mathfrak{k}$, then $\sum_{j=1}^{d}\left(d \pi\left(e_{j}\right)\right)^{2}$ is in the center of $\operatorname{End}\left(V_{\pi}\right)$ because \langle\rangle is $A d K$ invariant. Hence

$$
\sum_{j=1}^{d}\left(d \pi\left(e_{j}\right)\right)^{2}=-\lambda_{\pi} I_{V_{\pi}}
$$

for some nonnegative number $\lambda_{\pi}$.

Theorem 4.6. Let $a>0$. Suppose that $\pi$ is an irreducible representation of $K$. Then the function $u(g)=\operatorname{tr}(\pi(g))$ is in $\mathcal{H}_{a}$ if and only if

$$
\lambda_{\pi}<a^{2} .
$$

Proof. Let $B_{j}=d \pi\left(e_{j}\right)$ for some orthonormal basis $e_{1}, \ldots, e_{d}$ of $\mathfrak{k}$. Then $\lambda_{\pi} I_{V_{\pi}}=$ $-\sum_{j=1}^{d} B_{j}^{2}=\sum_{j=1}^{d} B_{j}^{*} B_{j}$. Fix $k$ in $K$ and consider the function $u_{k}(g):=u\left(k^{-1} g\right)$. Then

$$
\left\langle D^{n} u_{k}(e), e_{j_{1}} \otimes \cdots \otimes e_{j_{n}}\right\rangle=\operatorname{tr}\left(\pi\left(k^{-1}\right) B_{j_{1}} \cdots B_{j_{n}}\right) .
$$

In particular, for $k=e$, we find

$$
\left\langle\alpha_{n}, e_{j_{1}} \otimes \cdots \otimes e_{j_{n}}\right\rangle=\operatorname{tr}\left(B_{j_{1}} \cdots B_{j_{n}}\right)
$$

where $\alpha_{n}=\left(D^{n} u\right)(e)$. Thus by Schwarz' inequality for the trace we have, for $n \geq 1$,

$$
\begin{aligned}
\left|\alpha_{n}\right|^{2} & =\sum_{j_{1}, \ldots, j_{n}=1}^{d}\left|\operatorname{tr}\left(B_{j_{1}} \cdots B_{j_{n}}\right)\right|^{2} \\
& \leq \sum_{j_{1}, \ldots, j_{n}} \operatorname{tr}\left(I_{V_{\pi}}\right) \operatorname{tr}\left(B_{j_{n}}^{*} \cdots B_{j_{1}}^{*} B_{j_{1}} \cdots B_{j_{n}}\right) \\
& =\left(\operatorname{dim} V_{\pi}\right)^{2}\left(\lambda_{\pi}\right)^{n} .
\end{aligned}
$$

This also holds for $n=0$ because $\alpha_{0}=u(e)=\operatorname{tr}\left(I_{\pi}\right)$. Hence

$$
\begin{aligned}
\|\alpha\|_{, a}^{2} & =a^{-2} \sum_{n=0}^{\infty}\left(\left|\alpha_{n}\right|^{2} / a^{2 n}\right) \\
& \leq\left(a^{-1} \operatorname{dim} V_{\pi}\right)^{2} \sum_{n=0}^{\infty}\left(\lambda_{\pi} / a^{2}\right)^{n}
\end{aligned}
$$

which is finite if $\lambda_{\pi}<a^{2}$. This proves the sufficiency of (4.9). 
To prove the necessity of (4.9) let $C$ be the constant in Equation (4.1). Then, using (4.10) and Theorem $3.7 a$ ), twice, we find

$$
\begin{aligned}
C\|\alpha\|_{, a}^{2} & =C \int_{G}|u(g)|^{2} r_{a}(g) d g \\
& \geq \int_{G}\left|u\left(k^{-1} g\right)\right|^{2} r_{a}(g) d g \\
& =\sum_{n=0}^{\infty}\left(a^{2}\right)^{-n-1} \sum_{j_{1}, \ldots, j_{n}=1}^{d}\left|\operatorname{tr}\left(\pi\left(k^{-1}\right) B_{j_{1}} \cdots B_{j_{n}}\right)\right|^{2}
\end{aligned}
$$

for all $k$ in $K$. For any operator $A$ in $\operatorname{End}\left(V_{\pi}\right)$ we have $\int_{K}\left|\operatorname{tr}\left(\pi\left(k^{-1}\right) A\right)\right|^{2} d k=$ $\left(\operatorname{dim} V_{\pi}\right)^{-1} \operatorname{tr}\left(A^{*} A\right)$. Thus integrating (4.11) over $K$ we find

$$
\begin{aligned}
C\|\alpha\|_{, a}^{2} & \geq \sum_{n=0}^{\infty}\left(a^{2}\right)^{-n-1} \sum_{j_{1}, \ldots, j_{n}=1}^{d}\left(\operatorname{dim} V_{\pi}\right)^{-1} \operatorname{tr}\left(B_{j_{n}}^{*} \cdots B_{j_{1}}^{*} B_{j_{1}} \cdots B_{j_{n}}\right) \\
& =a^{-2} \sum_{n=0}^{\infty}\left(\lambda_{\pi} / a^{2}\right)^{n} .
\end{aligned}
$$

This proves the necessity of (4.9). Our proof also yields the estimates

$$
C^{-1} a^{-2}\left(1-\lambda_{\pi} / a^{2}\right)^{-1} \leq\|u\|_{, a}^{2} \leq\left(a^{-1} \operatorname{dim} V_{\pi}\right)^{2}\left(1-\lambda_{\pi} / a^{2}\right)^{-1} \text { for } \lambda_{\pi}<a^{2} .
$$

Q.E.D.

The following theorem is a kind of "local" Peter-Weyl theorem in that, as $a$ increases through any bounded interval, $\mathcal{H}_{a}$ enlarges by "adding on" the matrix elements of a finite number of irreducible unitary representations of $K$.

Theorem 4.7. A function $u$ is in $\mathcal{H}_{a}$ if and only if its Fourier series has the form

$$
u(k)=\sum_{\pi \in \widehat{K}, \lambda_{\pi}<a^{2}} \operatorname{tr}\left(\pi(k) A_{\pi}\right) .
$$

In particular

$$
\operatorname{dim} \mathcal{H}_{a}=\sum_{\lambda_{\pi}<a^{2}}\left(\operatorname{dim} V_{\pi}\right)^{2}
$$

which is finite.

Proof. If $u \in \mathcal{H}_{a}$, then Corollaries 4.3, 4.4 and Theorem 4.6 show that the only possible nonzero terms in its Fourier series (4.6) are those that appear in (4.13). Conversely, each term on the right in (4.13) is in $\mathcal{H}_{a}$ by Corollary 4.4 and Theorem 4.6. But the number of terms in (4.13) is finite. (See for example the discussion in Section 4 of $[\mathrm{H}]$. The argument given in the proof of Lemma 6 (i) in $[\mathrm{H}]$ applies to any $A d K$ invariant inner product on $\mathfrak{k}$.) Therefore the sum is in $\mathcal{H}_{a}$. Q.E.D.

Proposition 4.8. Let $\pi: K \rightarrow \operatorname{End}\left(V_{\pi}\right)$ be an irreducible unitary representation of $K$ and $d \pi$ be its infinitesimal representation of $\mathfrak{k}$. Let

$$
b=\sup \{\|d \pi(\xi)\|:|\xi| \leq 1, \xi \in \mathfrak{k}\} .
$$


Suppose that $A$ is in $\operatorname{End}\left(V_{\pi}\right)$ and $A \neq 0$. Let $u(g)=\operatorname{tr}(\pi(g) A)$ for $g$ in $G$. Then there exist strictly positive constants $C_{3}, C_{4}$ and $R$ such that

$$
C_{3} e^{b r} \leq \sup \{|u(g)|: g \in G,|g| \leq r\} \leq C_{4} e^{b r} \quad \text { if } \quad r \geq R .
$$

Proof. Since $V_{\pi}$ is finite dimensional, $\|d \pi(\xi)\|$ is continuous in $\xi$ on $\mathfrak{k}$. Therefore there exists a vector $\xi_{0}$ in $\mathfrak{k}$ such that $\left|\xi_{0}\right|=1$ and $\left\|d \pi\left(\xi_{0}\right)\right\|=b$. By replacing $\xi_{0}$ by $-\xi_{0}$, if necessary, we may assume that $b$ is an eigenvalue of the Hermitian operator $i d \pi\left(\xi_{0}\right)$. Let $i d \pi\left(\xi_{0}\right)=\sum_{j=1}^{m} \lambda_{j} P_{j}$ be the spectral resolution of $i d \pi\left(\xi_{0}\right)$ with $\lambda_{1}=b$. Then $\lambda_{j}<b$ for $j=2, \ldots, m$. For any $k_{1}$ and $k_{2}$ in $K$ we may write

$$
\begin{aligned}
u\left(k_{2}^{-1} \pi\left(e^{s i \xi_{0}}\right) k_{1}\right) & =\operatorname{tr}\left(e^{s i d \pi\left(\xi_{0}\right)} \pi\left(k_{1}\right) A \pi\left(k_{2}^{-1}\right)\right) \\
& =\sum_{j=1}^{m} e^{s \lambda_{j}} \operatorname{tr}\left(P_{j} \pi\left(k_{1}\right) A \pi\left(k_{2}^{-1}\right)\right) .
\end{aligned}
$$

Since $A \neq 0$ and $\pi$ is irreducible, $\left\{\pi\left(k_{1}\right) A \pi\left(k_{2}^{-1}\right): k_{1}, k_{2} \in K\right\}$ is fundamental in $\operatorname{End}\left(V_{\pi}\right)$ (as already noted in the proof of Corollary 4.4). Therefore there exists $k_{1}$ and $k_{2}$ in $K$ such that $\operatorname{tr}\left(P_{1} \pi\left(k_{1}\right) A \pi\left(k_{2}^{-1}\right)\right) \neq 0$. For such a choice of $k_{1}$ and $k_{2}$ we have

$$
u\left(k_{2}^{-1} \pi\left(e^{s i \xi_{0}}\right) k_{1}\right)=C_{1} e^{s b}+\sum_{j \geq 2} C_{j} e^{s \lambda_{j}}
$$

where $C_{1} \neq 0$. Consider only $s \geq 0$. Now $\left|i \xi_{0}\right|=1$. So $\operatorname{dist}\left(e, e^{s i \xi_{0}}\right) \leq s$. Therefore $\left|k_{2}^{-1} e^{s i \xi_{0}} k_{1}\right| \leq\left|k_{2}^{-1}\right|+s+\left|k_{1}\right|=s+c$, say. Then

$$
\sup _{|g| \leq s+c}|u(g)| \geq e^{s b}\left|C_{1}+\sum_{j=2}^{m} C_{j} e^{s\left(\lambda_{j}-b\right)}\right| .
$$

Since $\lambda_{j}-b<0$ for $j=2, \ldots, m$ there is real number $R$ such that the absolute value on the right of (4.10) is at least $\left|C_{1}\right| / 2$ when $s+c \geq R$. Writing $r=s+c$ we therefore have $\sup _{|g| \leq r}|u(g)| \geq e^{r b} e^{-c b}\left|C_{1}\right| / 2$ when $r \geq R$. This proves the first inequality in (4.16).

To prove the other half of (4.16) observe first that if $\zeta=\xi+i \eta$ with $\xi$ and $\eta$ in $\mathfrak{k}$, and if $X=d \pi(\xi)$ and $Y=d \pi(\eta)$ then, since $X$ and $Y$ are skew Hermitian, we have $\left\|e^{X+i Y}\right\| \leq\left\|e^{i Y}\right\|$ (by the Trotter product formula), which in turn is at most $e^{\|Y\|}$. But $\|Y\| \leq b|\eta|$ by (4.15). Since $|\eta| \leq|\zeta|$ we have $\left\|e^{d \pi(\zeta)}\right\| \leq e^{b|\zeta|}$. Now suppose that $\sigma:[0,1] \rightarrow G$ is an infinitely differentiable curve with $\sigma(0)=e$ and $\sigma(1)=g$. Let $c(s)=\left(L_{\sigma(s)^{-1}}\right)_{*} \sigma^{\prime}(s)$ where $\sigma^{\prime}(s)$ is the tangent vector to $\sigma$ at $s$. Let $w(s)=\pi(\sigma(s))$. Then $w(s)$ is the solution to the initial value problem $d w(s) / d s=w(s) d \pi(c(s)), w(0)=I_{V_{\pi}}$. We may represent the solution by Euler's method as a norm limit of products $w_{n}(1)=e^{d \pi c(0) / n} e^{d \pi c(1 / n) / n} \cdots e^{d \pi c((n-1) / n) / n}$. By the previous norm estimates we have $\left\|w_{n}(1)\right\| \leq \exp \left\{b \sum_{j=0}^{n-1}|c(j / n)| / n\right\}$. So $|w(1)| \leq \exp \left\{b \int_{0}^{1}|c(s)| d s\right\}=\exp \{b \cdot$ length of $\sigma\}$. Taking the inf over all such curves joining $e$ to $g$ we find $\|\pi(g)\| \leq e^{b|g|}$. Hence $|u(g)| \leq e^{b|g|} \operatorname{tr}\left\{\left(A^{*} A\right)^{1 / 2}\right\}$. Q.E.D.

Remark 4.9. Theorem 4.7 carries some interesting implications for norms on the universal enveloping algebra of $\mathfrak{k}$. Denote by $T_{r}$ the tensor algebra over $\mathfrak{k}$ and by $J_{r}$ the 2 -sided ideal in $T_{r}$ generated by $\{\xi \otimes \eta-\eta \otimes \xi-[\xi, \eta]: \xi, \eta \in \mathfrak{k}\}$. The spaces 
denoted by $T(\mathfrak{g})$ and $J$ in Sections 2 and 3 are the respective complexifications of $T_{r}$ and $J_{r}$. Any norm, \|\| , on $T_{r}$ induces a seminorm on the universal enveloping algebra $U:=T_{r} / J_{r}$ by the usual defintion: $\left\|\beta+J_{r}\right\|_{*}=\inf \left\{\|\beta+j\|: j \in J_{r}\right\}$. Write $\bar{T}_{r}$ for the completion of $T_{r}$ in the given norm, \|\| , and $\bar{J}_{r}$ for the closure of $J_{r}$ in $\bar{T}_{r}$. The map $\beta+J_{r} \rightarrow \beta+\bar{J}_{r}$ from $U$ into $\bar{T}_{r} / \bar{J}_{r}$ is clearly injective if and only if

$$
T_{r} \cap \bar{J}_{r}=J_{r}
$$

Moreover the kernel of this map is also the kernel of the seminorm \|\|$_{*}$. Thus the seminorm \|\|$_{*}$ is a norm if and only if (4.18) holds.

The family of norms (3.14) for $a>0$ and the family of norms dual to (2.9) for $t>0$ are of particular interest because of their role in heat kernel analysis on Lie groups. For the latter family of norms the identity (4.18) was conjectured in [G] and proven by $\mathrm{O}$. Hijab [Hi] when $\mathfrak{k}$ is the Lie algebra of a compact group. However for the former family of norms Theorem 4.7 implies that (4.18) must fail. Indeed Theorem 4.7 shows that $J_{, a}^{0}$ is finite dimensional over $\mathbb{C}$. Its "real" subspace $J_{, a}^{0} \cap T_{r}^{\prime}$ is therefore finite dimensional over $\mathbb{R}$. But the natural Hilbert space isomorphism of $\bar{T}_{r}$ onto its dual space $\left\{\alpha \in T_{r}^{\prime}:\|\alpha\|_{, a}<\infty\right\}$, in the pairing (2.4), clearly carries $\bar{T}_{r} \ominus \bar{J}_{r}$ onto $J_{, a}^{0} \cap T_{r}^{\prime}$. Hence $\bar{T}_{r} \ominus \bar{J}_{r}$ is finite dimensional. Therefore the map $\beta+J_{r} \rightarrow \beta+\bar{J}_{r}$ from the infinite dimensional space $T_{r} / J_{r}$ into the finite dimensional space $\bar{T}_{r} / \bar{J}_{r}$ cannot be injective. Thus (4.18) fails in a strong way for the norms (3.14).

\section{Example: $G=S L(2, \mathbb{C})$}

With this choice of $G$ we may take $K=S U(2)$. Then $G$ is the complexification of $K$. It will be shown that $\mathcal{H}_{a}(G)$ is finite dimensional and its dimension will be computed. As usual one can identify $\mathfrak{k}$ with $s u(2)$. Define

$$
\langle\xi, \eta\rangle=2 \operatorname{trace}\left(\eta^{*} \xi\right), \quad \xi, \eta \in s u(2) .
$$

This inner product is $A d K$ invariant. So we can apply the results of the preceding two sections. Let $\sigma_{3}=(i / 2) \operatorname{diag}(1,-1)$. Then $\sigma_{3}$ is in $s u(2)$ and is a unit vector with respect to the inner product (5.1). As is well known, the irreducible unitary representations of $S U(2)$ are parametrized by their spin $s \in\{n / 2: n=0,1,2, \ldots\}$. The representation $\pi_{s}$ of spin $s$ is of dimension $(2 s+1)$ and $\lambda_{\pi_{s}}=s(s+1)$. Furthermore $i d \pi_{s}\left(\sigma_{3}\right)$ can be diagonalized with diagonal entries $(s, s-1, \ldots,-s)$. In fact this holds for $i d \pi_{s}(\xi)$ for any unit vector $\xi$. Thus the constant $b$ in Equation (4.15) is $s$. Therefore, if $0 \neq A \in \operatorname{End}\left(V_{\pi_{s}}\right)$, then by Proposition 4.8 there are nonzero constants $C_{3}$ and $C_{4}$ such that

$$
C_{3} e^{s r} \leq \sup \left\{\left|\operatorname{tr}\left(\pi_{s}(g) A\right)\right|: g \in S L(2, \mathbb{C}),|g| \leq r\right\} \leq C_{4} e^{s r} .
$$

Proposition 5.1. Suppose that $u \in \mathcal{H}_{a}(S L(2, \mathbb{C}))$. Then $u$ has a finite Fourier decomposition of the form

$$
u(g)=\sum_{s(s+1)<a^{2}} \operatorname{tr}\left(\pi_{s}(g) A_{s}\right) .
$$

Any such function $u$ is in $\mathcal{H}_{a}$. In particular

$$
\operatorname{dim} J_{, a}^{0}=\operatorname{dim} \mathcal{H}_{a}=\sum_{s(s+1)<a^{2}}(2 s+1)^{2} .
$$


Proof. This is a special case of Theorem 4.7. Q.E.D.

\section{REFERENCES}

[B] V. Bargmann, On a Hilbert space of analytic functions and an associated integral transform. Part I, Communications on Pure and Applied Mathematics 14 (1961), 187-214. MR 28:486

[BO] C. Bender and S. Orszag, Advanced Mathematical Methods for Scientists and Engineers, McGraw Hill, 1978. MR 80d:00030

[D] B. K. Driver, On the Kakutani-Itô-Segal-Gross and the Segal-Bargmann-Hall isomorphisms, J. of Funct. Anal. 133 (1995), 69-128. MR 97j:22020

[DG] B. K. Driver and L. Gross, Hilbert spaces of holomorphic functions on complex Lie groups, Proceedings of the 1994 Taniguchi Symposium (to appear).

[G] L. Gross, The homogeneous chaos over compact Lie groups in Stochastic Processes, A Festschrift in Honor of Gopinath Kallianpur, S. Cambanis et al., Eds., Springer-Verlag, New York, 1993, pp. 117-123. MR 97j:22021

[H] B. Hall, The Segal-Bargmann "coherent state" transform for compact Lie groups, J. of Funct. Anal. 122 (1994), 103-151. MR 95e:22020

[Hi] Omar Hijab, Hermite functions on compact Lie groups. I, J. of Funct. Anal. 125 (1994), 480-492. MR 96e:22017

[K1] Paul Krée, Solutions faibles d'equations aux dérivées fonctionelles, Seminar Pierre Lelong I (1972/1973), in Lecture Notes in Mathematics, (See especially Sec.3), Vol. 410, Springer, New York/Berlin, 1974, pp.142-180. MR 51:8818

[K2] Paul Krée, Solutions faibles d'equations aux dérivées fonctionelles, Seminar Pierre Lelong II (1973/1974), in Lecture Notes in Mathematics, (See especially Sec.5), Vol. 474, Springer, New York/Berlin, 1975, pp.16-47. MR 52:14998

[K3] Paul Krée, Calcul d'intégrales et de dérivées en dimension infinie, J. of Funct. Anal. 31 (1979), 150-186. MR 80k:46051

[S1] I. E. Segal, Tensor algebras over Hilbert spaces, Trans. Amer. Math. Soc. 81 (1956), 106134. MR 17:880d

[S2] I. E. Segal, Mathematical characterization of the physical vacuum for a linear Bose-Einstein field, Illinois J. Math. 6 (1962), 500-523. MR 26:1075

[S3] I. E. Segal, The complex wave representation of the free Boson field, in "Topics in functional analysis: essays dedicated to M. G. Krein on the occasion of his 70th birthday", Advances in Mathematics: Supplementary studies, Vol. 3 (I. Gohberg and M. Kac, Eds.), Academic Press, New York, 1978, pp. 321-344. MR 82d:81069

Department of Mathematics, Cornell University, Ithaca, New York 14853

E-mail address: gross@math.cornell.edu 